\title{
Possible Future Applications of Photonic Bandgap Fiber in Non-Repeatered Transmission
}

Kazunori Mukasa, Francesco Poletti, Marco Petrovich, Neil Broderick, R. Amezcua-Correa, M.A.F.Roelens and D.J. Richardson

\author{
U niversity of Southampton, 0 ptoelectronics Research Centre (ORC) \\ Highfield, Southampton, Hampshire, SO 17 1BJ , U.K. \\ Tel +44(0)2380593139 Fax +44(0)238059 3142 e-mail mukasa@ ch.furukawa.co.jp
}

\begin{abstract}
We investigate the potential use of photonic bandgap fibers in non repeatered transmission systems. Our simulations show that significant improvements in system reach should be possible as a result of the reduced nonlinearity providing that the fiber losses can be reduced to the 0.2 to $0.3 \mathrm{~dB} / \mathrm{km}$ regime.
\end{abstract}

\section{Introduction}

As a result of steady technological improvements, particularly in the development of low-loss, large-mode-area, pure silica core fiber (LMA-PSCF), and the use of remotely pumped/distributed amplifiers, the maximum achievable transmission distances within Non-Repeatered Transmission Systems (NRTS) have now been pushed beyond $500 \mathrm{~km}$, making NRT an interesting proposition for the implementation of low cost submarine systems [1]. The maximum transmission distance is now limited primarily by nonlinear effects which restrict both the amount of signal power that can be launched at the transmitter end, and the amount of power that can be launched at the receiver end to pump a remote amplifier. Consequently, any fiber that permits significantly enhanced levels of power to be transmitted without nonlinear distortion is of great interest for the further development of NRTS. Hollow Core Photonic Band Gap Fiber (HC-PBGF) technology offers just this as it enables fibers with one to two orders of magnitude less nonlinearity per unit length than PSCF. Moreover, it may also ultimately offer the possibility of lower transmission losses [2]. Assessing the potential impact of HC-PBGF fibers in NRT thus appears a topic worthy of consideration.

In this paper we present the design of a HC-PBGF that offers a low nonlinearity per unit length, and both dispersion and confinement loss characteristics within the C-band suited for NRTS applications. We consider two possible application scenarios. Firstly, we consider use of this fiber in a NRTS operating at the fiber's zero-dispersion wavelength, and compare its performance to that of a system based on conventional dispersion shifted fiber types (i.e. DSF and NZ-DSF). Secondly, we consider the possibility of incorporating a length of HC-PBGF at the transmitter end of a transmission line composed otherwise of PSCF. This approach reduces the nonlinearity where the power levels are the highest whilst exploiting the low loss features of PSCF in the remainder of the system.

\section{The design of the dispersion shifted PBGFs (DS-PBGFs)}

We used Finite Element Method (FEM) based calculations to design a fiber suited for NRT. We focused on fiber designs according to the hexagonal structure shown inset in Fig.1, (and described more fully in Ref [3]), which we have previously shown to offer broadband, surface-mode free transmission, and a low nonlinearity per unit length. We adopted a design with a 19-cell defect core and 7 layers of air holes within the cladding. For a d/ $\Lambda$ of $\sim 0.97$ and $\Lambda=4.33 \mu \mathrm{m}$ we obtained a fiber with a band gap centered at $1650 \mathrm{~nm}$ for which the overlap of the fundamental mode and air is $99.3 \%$, and $\lambda 0$ for the fundamental mode is at $1.56 \mu \mathrm{m}$. Using the definitions proposed in Ref. [4], the effective mode area and the effective nonlinear refractive index were estimated to be $138 \mu \mathrm{m}^{2}$ and $3.08 \times 10^{-23} \mathrm{~m}^{2} / \mathrm{W}$ respectively, corresponding to $\gamma=0.9 \times 10^{-3}(\mathrm{Wm})^{-1}$.
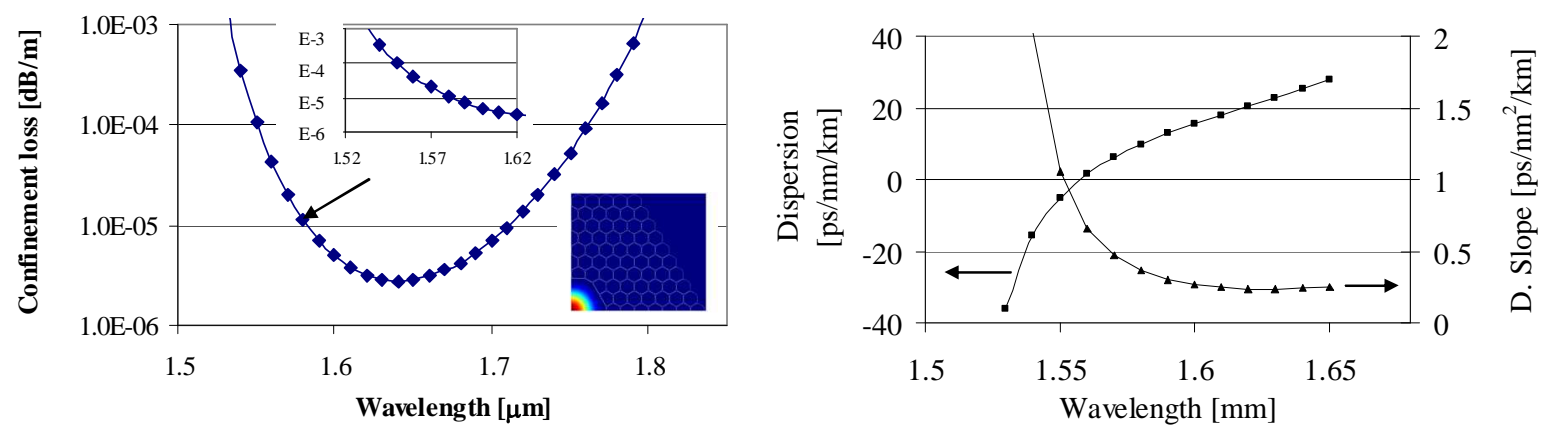

Fig.1 The simulation results of DS-PBGF that has $\lambda_{0}$ at 1560nm (Left: confinement loss, Right; Dispersion property) 
The dispersion of this fiber was $-5.13 \mathrm{ps} / \mathrm{nm} / \mathrm{km}$ at $1550 \mathrm{~nm}$ meaning that it could be used as negative-dispersion NZDSF at this wavelength, although it is to be appreciated that the dispersion slope is very large $\left(1.056 \mathrm{ps} / \mathrm{nm}^{2} / \mathrm{km}\right)$. This slope would need to be compensated for WDM applications. The predicted confinement losses were $0.11 \mathrm{~dB} / \mathrm{km}$ at $1550 \mathrm{~nm}$ and $0.04 \mathrm{~dB} / \mathrm{km}$ at $1560 \mathrm{~nm}$, although these losses could be further reduced with the incorporation of an additional ring of cladding holes. The fiber is capable of supporting higher order modes however these have a far higher confinement loss and consequently we consider only the fundamental mode in the calculations that follow. Furthermore we neglect the impact of PMD and multi-path interference.

\section{Single channel non-repeater transmission over $\lambda 0$ of DSF, NZ-DSF and PB GF}

We evaluated the system performance of this fiber for both $40 \mathrm{~Gb} / \mathrm{s}$ and $80 \mathrm{~Gb} / \mathrm{s}$ single channel CS-RZ transmission whilst operating at the zero dispersion wavelength at $1560 \mathrm{~nm}$, and compared this performance with that of conventional DSF and high performance NZ-DSF (NZ-DSF) [5]. We used commercial $\mathrm{VPI}^{\mathrm{R}}$ software for the simulations and performed calculations for PBGF losses of both $0.30 \mathrm{~dB} / \mathrm{km}$ and $0.22 \mathrm{~dB} / \mathrm{km}$. The latter loss value corresponds to the estimated minimum loss resulting from capillary wave formation as predicted in Ref [2]. In all of our simulations the input power to the fiber was optimized to obtain the lowest possible Power Penalty (PP). The performance of the three different transmission lines was evaluated in terms of the power penalty at a BER of $10^{-9}$ relative to the back-to-back case. A PP of $2 \mathrm{~dB}$ was used as a criterion to define the maximum transmission length. The simulated transmission results are summarized in Table 1 and Fig.3. It is seen that the PBGFs support the longest transmission lengths for both $40 \mathrm{~Gb} / \mathrm{s}$ and $80 \mathrm{~Gb} / \mathrm{s}$ as a result of the low fiber nonlinearity. However, it is to be emphasized that maintaining a low fiber loss as well as nonlinearity is essential since for the $0.3 \mathrm{~dB} / \mathrm{km}$ case the power levels required at the input approach the $\mathrm{kW}$ regime - which is obviously far from practical. However, these simulations serve to highlight the level of nonlinearity reduction associated with these fibers.

Table 1 Optical properties of the fibers used and system results in terms of maximum transmission distance

\begin{tabular}{|l|l|l|l|l|l|l|l|}
\hline Type & Loss & Dispersion & Slope & Aeff & $\mathrm{n} 2$ & $40 \mathrm{~Gb} / \mathrm{s}$ & $80 \mathrm{~Gb} / \mathrm{s}$ \\
\hline & $\mathrm{dB} / \mathrm{km}$ & $\mathrm{ps} / \mathrm{nm} / \mathrm{km}$ & $\mathrm{ps} / \mathrm{nm}^{2} / \mathrm{km}$ & $\mu \mathrm{m}^{2}$ & $\mathrm{~m}^{2} / \mathrm{W}$ & $\mathrm{km}$ & $\mathrm{km}$ \\
\hline C-DSF & 0.20 & 0 & 0.070 & 45 & $2.7 \mathrm{E}-20$ & 170 & 120 \\
\hline NZ-DSF & 0.21 & 0 & 0.100 & 85 & $2.7 \mathrm{E}-20$ & 200 & 160 \\
\hline PBGF-1 & 0.30 & 0 & 0.600 & 138 & $3.08 \mathrm{E}-23$ & 250 & 200 \\
\hline PBGF-2 & 0.22 & 0 & 0.600 & 138 & $3.08 \mathrm{E}-23$ & 330 & 300 \\
\hline
\end{tabular}

$\begin{array}{lll}\text { Tx } 1 & \text { Pre-EDFA } & \\ \text { AdB }-1 \mathrm{THz} & & \text { Total A dB loss } \\ 40 \mathrm{~Gb} / \mathrm{s} & 5 \mathrm{~dB} \mathrm{NF} & \text { Dispersion }=0\end{array}$

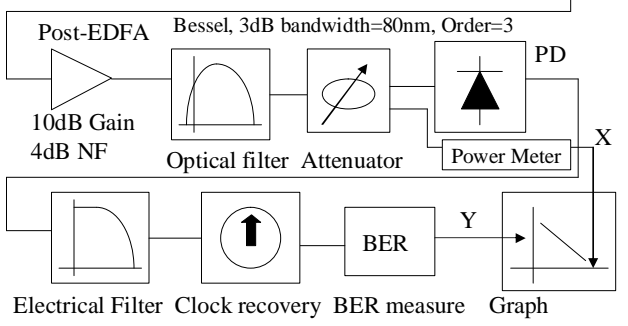

Fig.2 Single channel non-repeatered transmission system (40Gb/s) using C-DSF, NZ-DSF and PBGFs

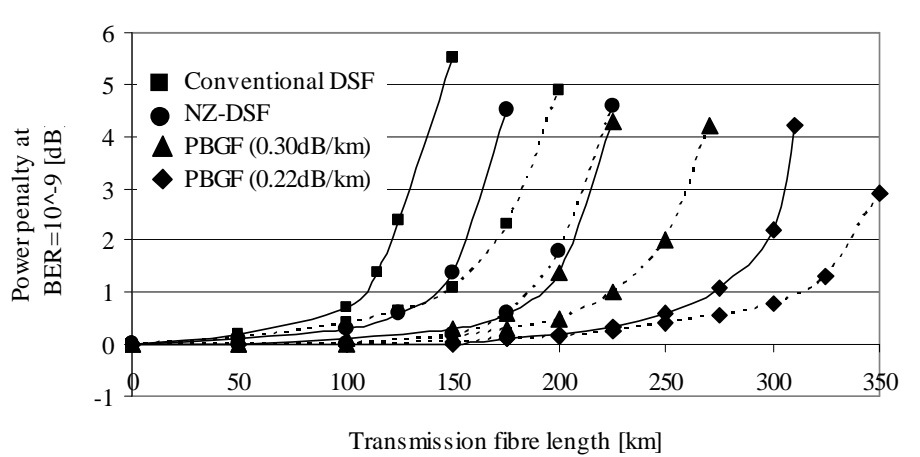

Fig.3 Single channel 40Gb/s (dotted line) and 80Gb/s (solid line) non repeatered transmission performances of the C-DSF, NZ-DSF and PBGFs $(0.30 \mathrm{~dB} / \mathrm{km}$ and $0.22 \mathrm{~dB} / \mathrm{km})$

\section{Single channel NRTS using PSCF +DCF or PBGF +PSCF +DCF}

We investigated the use of PBGFs in conjunction with PSCF in a single channel $10 \mathrm{Gbit} / \mathrm{s}$ CS-RZ NRTS. PSCF offers the current best solution in terms of providing the lowest fiber losses. However, these fibers exhibit relatively large dispersion within the C-band and thus dispersion compensation using a high performance dispersion compensating fiber (HP-DCF) is required. We first evaluated the performance of two PSCF NRTS each of a total length of $300 \mathrm{~km}$ comprising differing specified lengths of PBGF at the transmitter end (and a suitable length of HP$\mathrm{DCF}$ at the receiver [6] as required to compensate the nett-dispersion and dispersion slope). We chose PBGF lengths of $10 \mathrm{~km}$ (referred to as NRT system TL-B) and $20 \mathrm{~km}$ (TL-C) for this study. We then compared the performance of 
these systems with that of a NRTS comprising a $300 \mathrm{~km}$ length of PSCF and an associated HP-DCF (TL-A.). In addition, we modeled a system incorporating a 50km length of PBGF inserted before the $300 \mathrm{~km}$ of PSCF providing a total transmission line length of $350 \mathrm{~km}$ (TL-D). The optical properties of the fibers used for the simulations are summarized in Table2, and the precise system configuration modeled is shown in Fig.4.

The input powers into the TL were again optimized to obtain the lowest PP. Fig.5 and Table 2 show the Bit Error Rate (BER) and PP evaluation results for the back-to-back, and for configurations TL-A through to TL-D. As shown in Fig.5 and Table2, use of both 10 or 20km of PBGFs at the input where the powers are highest, drastically improves the BER performance due to the reduced nonlinearity in this part of the system. Importantly, note that no BER degradation was observed by adding $50 \mathrm{~km}$ PBGF to TL-A (case TL-D). To further quantify the merit of using the PBGF at the input to the TL we estimated the total peak nonlinear phase shift for each NRTS considered. If we assign a normalized nonlinear phase shift of 1 for TL-A then the relative phase shifts were $0.603,0.364$ and 0.804 for TL-B, TL-C and TL-D respectively. Note that for TL-A, B and C, the input powers were typically a few W, whereas the values for TLD was of order $15 \mathrm{~W}$ as shown in Table 2- an entirely feasible value given recent advances in high power fiber amplifier technology. Note that although these calculations were for a single channel the same approach should be applicable to WDM NRT [2] due to the compensated dispersion.

Table 2 Optical properties of the PBGF, PSCF and DCFs and power penalties obtained for each combination.

\begin{tabular}{|c|c|c|c|c|c|c|c|}
\hline & Loss & Dispersion & Slope & Aeff & $\mathrm{n} 2$ & \multirow{2}{*}{$\begin{array}{l}\text { Configuration } \\
\text { (Input Power) }\end{array}$} & \multirow{2}{*}{$\begin{array}{l}\text { PP at BER10 } \\
\text { (Received sensitivity) }\end{array}$} \\
\hline & $\mathrm{dB} / \mathrm{km}$ & $\mathrm{ps} / \mathrm{nm} / \mathrm{km}$ & $\mathrm{ps} / \mathrm{nm}^{2} / \mathrm{km}$ & $\mu \mathrm{m}^{2}$ & $\mathrm{~m}^{2} / \mathrm{W}$ & & \\
\hline PBGF & 0.22 & -5 & 1.000 & 138 & $3.08 \times 10^{-23}$ & TL-B $(2.83 \mathrm{~W})$ & $1.0(-30.0 \mathrm{dBm})$ \\
\hline PSCF & 0.17 & 20 & 0.060 & 110 & $2.2 \times 10^{-20}$ & TL-C $(3.17 \mathrm{~W})$ & $0.4(-30.6 \mathrm{dBm})$ \\
\hline $\mathrm{DCF}$ & 0.60 & -250 & Adjustable & 15 & $4.0 \times 10^{-20}$ & TL-D $(15.84 \mathrm{~W})$ & $2.8(-28.2 \mathrm{dBm})$ \\
\hline
\end{tabular}

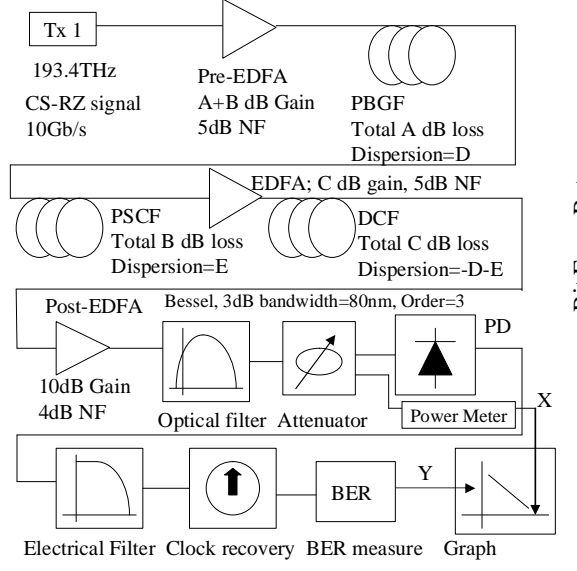

Fig.4 Single channel non-repeatered transmission system $(10 \mathrm{~Gb} / \mathrm{s})$ using PBGFs and PSCFs + DCFs

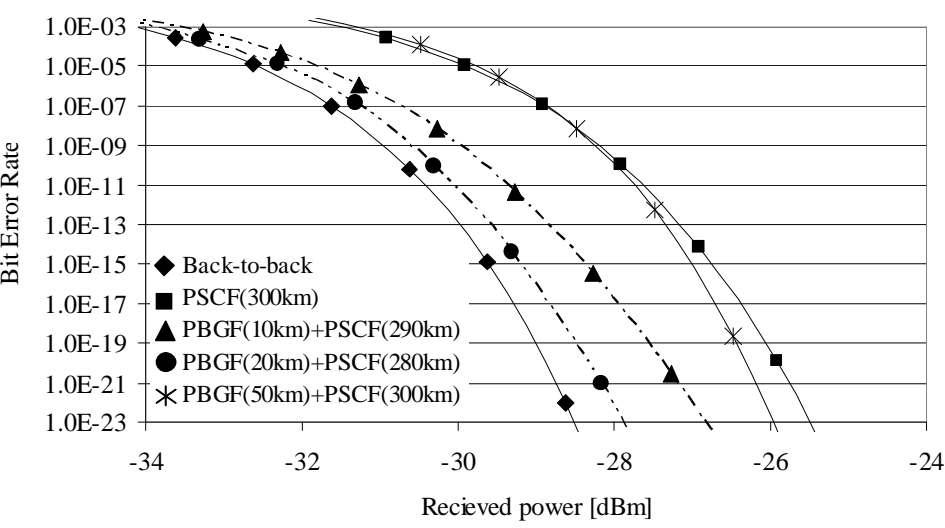

Fig.5 Single channel non-repeatered transmission BER evaluation results of back-to-back, PSCF $(300 \mathrm{~km})$ and PBGFs $(10 \mathrm{~km}, 20 \mathrm{~km}$ and $50 \mathrm{~km})+$ PSCFs + DCFs

\section{Conclusion}

In conclusion we have investigated the potential use of PBGFs for extending the reach of an NRTS. By strategically incorporating these low nonlinearity fibers within an NRTS to increase the usable signal power level it should be possible to extend the reach of both single channel and WDM systems. Moreover, it should also be possible to apply the same principle at the receiver end of a remotely pumped NRTS in order to extend the attainable pump reach of the system. Spans well in excess of $600 \mathrm{~km}$ should theoretically be achievable using these ideas although clearly major technological/fabrication challenges will need to be overcome if this is ever to be realised in practice.

\section{R eferences}

[1] L.Labrunie et al., Electronics Let., Vol.39, No.19, September, pp.1394-1395, (2003)

[2] P. J. Roberts et al., Opt. Express, Vol. 13, No.1, pp.236-244 (2005)

[3] R. Amezcua-Correa et al., Opt. Express, Vol. 14, No.17, pp. 7974-7985 (2006)

[4] R. Heinberger et al., IEEE Photon. Tech. Lett., Vol. 17, No.1, pp.70-71, (2005)

[5] K.Mukasa et al., Proceeding of OECC'98, 15C1-4, pp.366-367, (1998)

[6] L.Gruner-Nielsen et al., Journal of Lightwave tech., Vol 23, No.11, November, pp.3566-3579, (2005) 